\title{
Assessment of production processes functioning in the case of air bag production
}

\author{
Krzysztof Mielczarek ${ }^{1, *}$, and Krzysztof Knop ${ }^{1}$ \\ ${ }^{1}$ Czestochowa University of Technology, Faculty of Management, Department of Production \\ Engineering and Safety, Armii Krajowej 19B, 42-200 Czestochowa, Poland
}

\begin{abstract}
In the chapter was presented enterprise producing air bags. described. Toyota's management principles by Jeffrey Liker were pointed out, which refers to production process (second). An innovative BOST questionnaire survey was characterized as a tool for transformation of Toyota's management principles into questions. Survey and research method determined as BOST (the name of the is an acronym created from the first two letters of the name and surname of his creator i.e. Stanislaw Borkowski, professor of technical and economic sciences, the acronym is legally protected). Results of the questionary were introduced. It was made an analysis in the range of the principle 2 of Toyota. It was shown analysis of respondent's characteristics. Next structure of assessments was determined and some statistical graphs were built.
\end{abstract}

\section{Introduction}

The air bag is a device of passive protecting passenger's compound from one or two pillows. In the case of a head-on collision are automatically inflated constituting the amortization between absorbing front seats of the driver and the passenger and with the steering wheel or the front part of the passenger cabin. The air bag consists of the generator of gas and the sack made of special material. The charge in the generator of gas is being initiated electrically, through impulse of sensors located in different places of the vehicle. A task of sensors is to recognize where the hitting was started and to activate appropriate air bags. As a result of the slight bump air bags cannot be started. After filling pillows, a gas immediately is escaping in specially prepared holes. Thanks to such a solution correct shock absorption is possible while the body is hitting the pillow. This solution prevents for strangulation of the person who e.g. lost consciousness. We are distinguishing the following kinds of air bags: frontal air bag, the side air bag, the knee air bag, air curtains and the air bag for the center passenger of the back couch. A production process, during the product is coming into existence, is a very essential stage. It decides about final evaluation taken out by the customer. Enterprise, which aspiring to the excellence of its product, realizes the necessity of constant improvement. The product should fulfil the highest requirements in terms of the functionality, the industrial safety and the environmental protection.

\footnotetext{
*Corresponding author: krzysztof.mielczarek@wz.pcz.pl
} 


\section{Presentation of respondent's structure}

The personnel rating are the best indicator of the correctness of individual areas in the enterprise. To the purpose of analyzing satisfying the staff, a hierarchy of importance of influencing factors to the correctness of action given area and raising the quality by the vision and satisfying in the company, a BOST questionnaire form was carried out by 30 workers from production department. Amongst selected enterprise the population of respondents was chosen, which consisted from production workers of the examined enterprise, having a contact with production process in the workplace. Studies were marked by a low degree of anonymity, but with large degree of control by the interviewer. Applied questionnaire form - lecture and direct questionnaire - affected on increase in measurement control. It was an intentional treatment, which was supposed to contribute in increase of the correctness in filling surveys by pollsters In Table 1 was presented characteristics of respondents in table set.

Table 1. Respondents features in researched company.

\begin{tabular}{|c|c|c|c|c|c|c|}
\hline & \multicolumn{6}{|c|}{ Feature indication and its characteristics } \\
\hline & Gender & Educations & Age & $\begin{array}{c}\text { Work } \\
\text { experience }\end{array}$ & Mobility & Way of employment \\
\hline 1 & Man & $<$ Average & $<30$ & $<5$ & 1 & Normal \\
\hline 2 & Woman & Average & $31-40$ & 6 to 10 & 2 & Transfer \\
\hline 3 & & Higher I & $41-50$ & 11 to 15 & 3 & Finance \\
\hline 4 & & Higher II & $51-55$ & 15 to 20 & 4 & \\
\hline 5 & & & $56-60$ & 21 to 25 & 5 & \\
\hline 6 & & & $61-65$ & 26 to 30 & 6 & \\
\hline 7 & & & $>66$ & 31 to 35 & & \\
\hline 8 & & & & $>36$ & & \\
\hline
\end{tabular}

On the basis of collected results circular graphs presenting participation of the given feature in the set (Fig. 1). From all respondents, man presents $67 \%$ of examined crew (Fig. 1a). The majority of respondents has the university education. In the case of age (Fig. 1c) the most workers were from 31 to 40 years (33\% of examined crew). The largest groups constituted persons with the work experience on level 6 - 10 years $(38 \%)$.

a

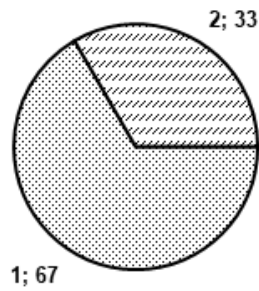

\section{c}

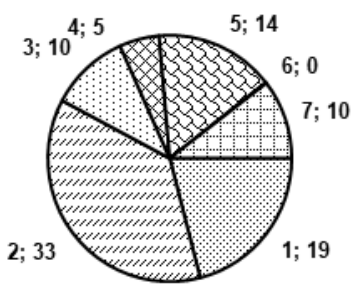

b.

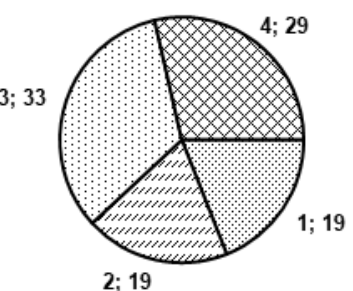

d

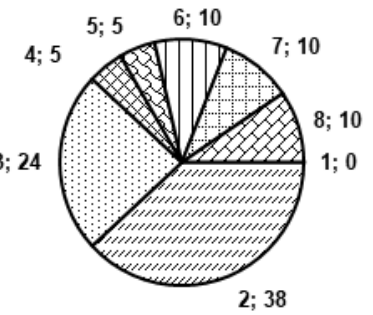



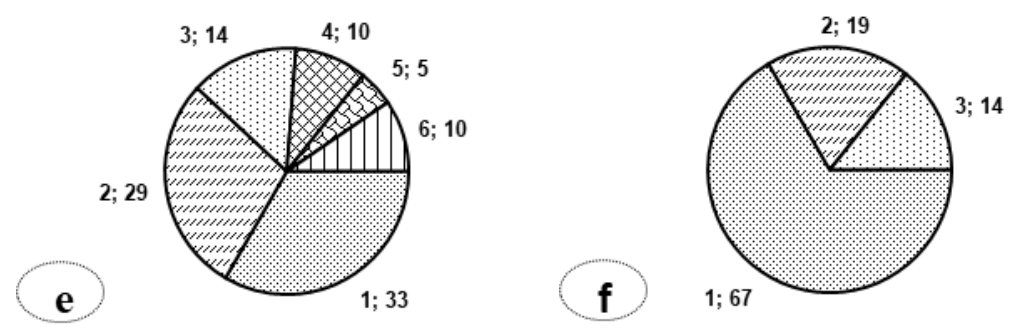

Fig. 1. Percentage (circular charts) characteristics of respondents with consideration: a) gender, b) educations, c) age, d) work experience, e) mobility, f) way of employment.

Analyzing the personal feature of respondents - mobility we observed that persons, for whom the current job is first constituted the majority of respondents. Analyzing way of recruitment (Fig. 1f) we noticed that persons with contract of employment constituted the largest group $-(67 \%$ of the whole $)$.

\section{An evaluation the production process factors according to the BOST method}

Toyotarity is a scientific discipline examining human - machine and human - human relationships with the inclusion of a process-based approach, Japanese culture, especially of Toyota, oriented to continuous improvement with the use of knowledge [1]. The BOST method describes Toyota's management principles with its characteristic factors. Valuable complement of carried out research there is interpretation of BOST questionnaire results [2]. The BOST method allows assessing the significance of factors describing the 14 Toyota management principles. It lets better look on the enterprise by eyes of their workers [3-4]. In the purpose to form an opinion it is essential to know the opinion of workers from different ranks in enterprise. BOST is survey where the questions are so well-matched to judge enterprise and its immaterial stores are possible. It was made on the base of 14 principles of the Toyota [5]. The second Toyota management principle is based on the conviction that appropriate process leads to appropriate results [6]. If the process is designed properly, then good results will come automatically [7]. It is necessary that quality is built in the processes already on the initial stage of production [8]. Processes shall be reorganized so that their course is fluent and uniform. Succeeding and improving competitiveness is only possible in self-learning organization and by continuous improvement of an organization culture [9].

Question that was analyzed sounded ,Which factor is the most important in the production process?" (fill the blanks with $1 ; 2 ; 3 ; 4 ; 5 ; 6 ; 6$ - the highest factor). The person who is filling questionnaire is judging the following factors:

- Continuous system of problem detection (CP).

- Stopping production after discovering a quality problem (PE).

- Standard tasks, processes, documents (SZ).

- Providing authorizations to subordinates (EU).

- Application of only reliable technology (ST).

- Application of visual control (SN).

The question has connection with 2 principle of the Toyota (E3 area). In Table 2 a percentage combination of evaluations was presented. 
Table 2. Percentage list [\%] of importance ratings for factors of E3 area.

\begin{tabular}{|c|c|c|c|c|c|c|}
\hline \multirow{2}{*}{ Evaluation } & \multicolumn{7}{|c|}{ Indicating the factors } \\
\cline { 2 - 7 } & CP & PE & SZ & EU & ST & SW \\
\hline 1 & 0.0 & 4.8 & 28.6 & 14.3 & 19.0 & 33.3 \\
\hline 2 & 0.0 & 28.6 & 9.5 & 38.1 & 9.5 & 14.3 \\
\hline 3 & 14.3 & 19.0 & 23.8 & 19.0 & 9.5 & 14.3 \\
\hline 4 & 19.0 & 23.8 & 23.8 & 14.3 & 14.3 & 4.8 \\
\hline 5 & 47.6 & 14.3 & 4.8 & 14.3 & 4.8 & 14.3 \\
\hline 6 & 19.0 & 9.5 & 9.5 & 0.0 & 42.9 & 19.0 \\
\hline
\end{tabular}

On the basis of the results presented in Table 2 the following circle charts have been created (Fig. 2).

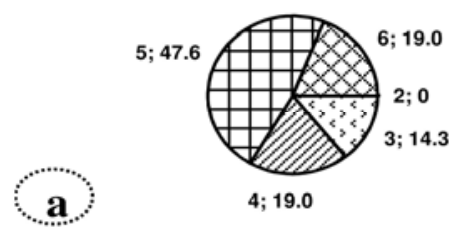

b)
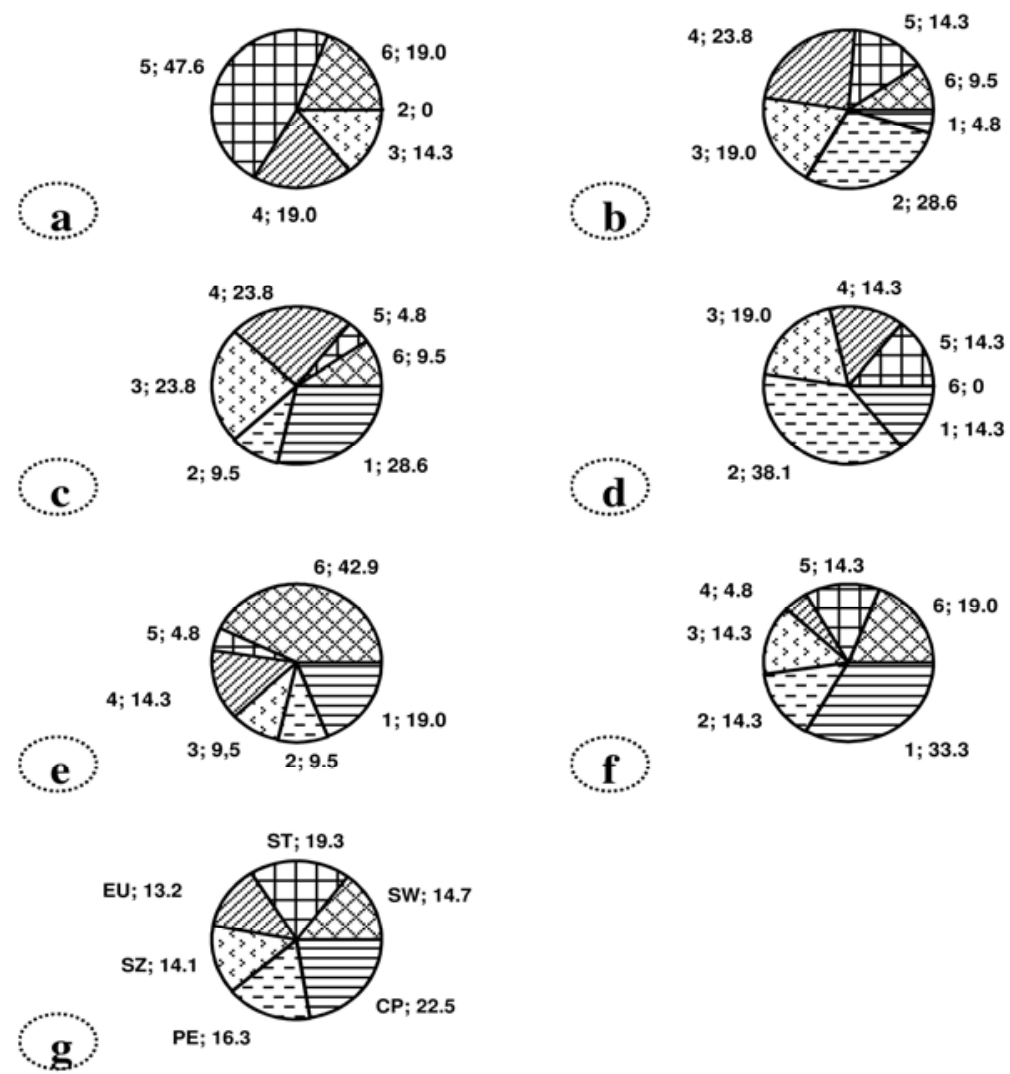

Fig. 2. Principle 2. Circle charts - structure for the ratings: a) CP, b) PE, c) SZ, d) EU, e) ST, f) SW, g) average.

Continuous system of problem detection (CP) according to $47.6 \%$ respondents has a deciding influence on the production process because they granted high rate " 5 ". On the basis of the prepared structure of ratings for separate factors it was found that Application of only reliable technology (ST) was the most important factor for $42.9 \%$ of respondents. They have awarded that factor the highest rate " 6 ". 
On the basis of acquired results the following significance sequences of factors describing the second Toyota management principles have been created in the production process of air bag:

$$
\mathrm{CP}>\mathrm{ST}>\mathrm{PE}>\mathrm{SZ}>\mathrm{SW}>\mathrm{EU}
$$

From analysis of the range of important factors it results that Providing authorizations to subordinates $(\mathrm{EU})$ is not a key factor of the production process (among examined factors it is on the last place in a range of important factors of the production process).

The majority of factors has similar values. For $22.5 \%$ of respondents Continuous system of problem detection (CP) has high meaning in the organization of the production system. In the case of the factor Application of only reliable technology (ST) reached average 19.3\%. According to respondents independence Providing authorizations to subordinates (EU) with the equal average $13.2 \%$ is the least important.

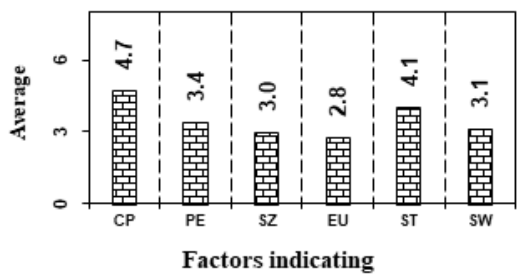

Fig. 3. Average (numerical) structure of the rating describing the second Toyota management principle.

To conclude it is possible, that workers are thinking highly of constant quality improvement, it just goes to show that this factor receiver high assessment. Assessments of remaining factors are distributed quite evenly. Respondents rank the factors in logical way and it proves proper approach of workers towards production process.

Radar graphs (Fig. 4) show importance depicting the percentage disintegration of factors describing the second Toyota management principle. On individual graphs they are arranged in the decreasing order according to the direction of movement of pointers of the clock having begun from hour 12:00, percentage shares of the assigned evaluations by respondents for each factor. Analysis of the relation showed that for some cases the equal percentage participation of factors had appeared. An occurrence of the change of the factor position in the importance series is also happening together with the height of a marking scale.
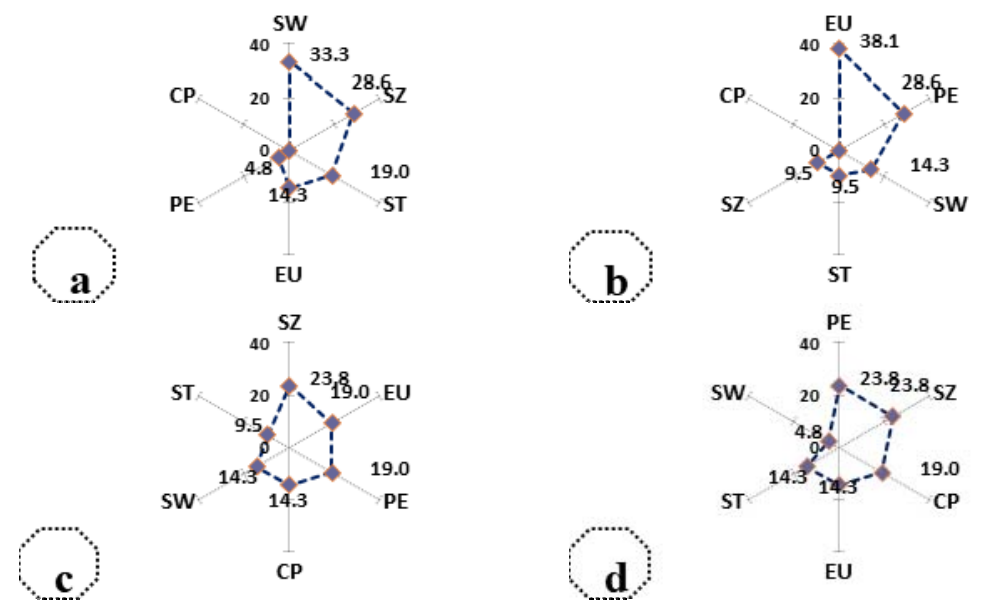


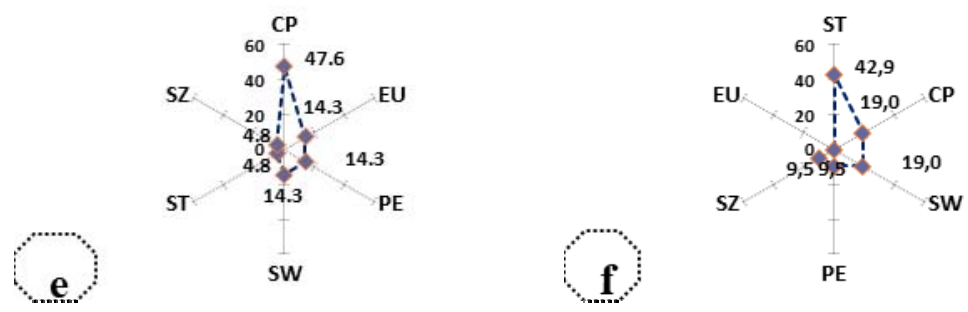

Fig. 4. Radar charts for importance factors of 2 principle of the Toyota: a) „1”, b) „2”, c) „3”, d) „4, e) ,5”, f), ,6".

Factor Continuous system of problem detection (CP) appeared on the position dominating only for evaluation „6". For the evaluation „1" and ,2” the factor took the last place.

\section{Summary}

The Toyota management principles which are associated with production process was presented. Innovative BOST questionnaire survey, which are an attempt to convert Toyota's management principles into questions was described. The starting point for changes (improvement) is recording the existing condition. The present situation is definitely known best by participants of the processes implemented in a given enterprise. The analysis of significance of factors describing the second Toyota management principle states that the factor Continuous system of problem detection (CP) is the most important. The second position was taken by the following factor - Application of only reliable technology (ST). These factors are closely connected with technological aspects of production relating to fluent production rhythm with simultaneous application of reliable methods and technologies. The analysis has revealed diversity in the significance of factors describing the second Toyota management principle. In this way the usefulness of the BOST method has been proved for assessing a production process of goods of high quality requirements.

\section{References}

1. J.K. Liker, The Toyota Way. 14 Principles of management of the world's leading manufacturing company (Publisher MT Business, Warsaw, 2005)

2. K. Amasaka, Science TQM, New Quality Management Principle, The Quality Management Strategy of Toyota Introduction (Bentham Sc., U Arab Emirates, 2012)

3. S. Borkowski, Toyota's management principles in the questions. The results of BOST (PTM Publishing House, Warsaw, 2012)

4. S. Borkowski, Scientific Potential of Toyotarity and BOST Method (Polish Quality Institute, Warsaw, 2016)

5. K. Knop, Production Engineering Archives, 7/2, 40-44, (2015)

6. S. Borkowski, J. Rosak-Szyrocka, D. Klimecka-Tatar, M. Jagusiak-Kocik, P. Sygut, $22^{\text {nd }}$ International Conference on Metallurgy and Materials METAL (Brno, 2013)

7. J. Selejdak, Production Engineering Archives, 1/1, 13-15 (2013)

8. M. Zhuravskaya, E. Morozova, N. Anashkina, M. Ingaldi, Polish Journal of Management Studies, 13/2, 192-203, (2016)

9. K. Mielczarek, Production Engineering Archives, 8/3, 32-3, (2015) 